\title{
Successful late repair of a post-traumatic bronchial stricture
}

\author{
H. VAN DER SCHAAR, J.P. M. WAGENAAR ${ }^{1}$, \\ J. SWIERENGA, and A. G. BROM \\ Department of Thoracic Surgery and Pulmonary Diseases, University Hospital, Leyden, the Netherlands
}

After a blunt thoracic injury a patient developed bronchial stenosis with post-stenotic infected bronchial dilatation. These changes proved to be reversible after resection of the bronchial stenosis.

In the past few years the risk of bronchial rupture following thoracic injury has been discussed in several papers (Truckenbrodt and Gall, 1968; Eijgelaar and Homan van der Heide, 1970). If complete bronchial rupture occurs, surgical therapy in the form of re-anastomosis must follow as soon as possible.

In the case of a partial bronchial rupture it is sufficient to institute conservative therapyantibiotics and drainage of the pleural cavity. However, there is the possibility of stenosis with post-stenotic atelectasis and bronchiectasis.

Isfort (1967) stated that this condition can occur within a few weeks or after several months. Once stenosis occurs, surgical intervention is necessary. The question whether surgical repair of the bronchus is useful once there is unmistakable evidence of post-stenotic bronchial changes has been insufficiently answered in the literature. Drapanas, Siewers, and Feist (1966) advised in favour of surgical repair of the bronchus, even in the presence of bronchiectatic changes, and presented a pertinent case report. They maintained that surgery is indicated if it can be carried out in an early post-traumatic stage when the bronchial changes are still reversible. Prompted by our own experience with such a case, we believe that surgical repair of the bronchus should be considered even after the occurrence of post-stenotic bronchial dilatation, with or without infection.

\section{CASE REPORT}

In February 1966, a 19-year-old girl was admitted to hospital elsewhere following a traffic accident; she was dyspnoeic and cyanotic when admitted, and examination disclosed subcutaneous emphysema of the 1Reprint requests to J. P. M. Wagenaar, M.D., Department of
Pulmonary Diseases, University Hospital, Leyden, The Netherlands cervical region and the chest wall. Further physical findings were negative apart from bruising. The chest radiograph showed partial left pneumothorax.

The patient was treated by intubation of the left pleural cavity and antibiotic medication, which initially seemed to be effective. A radiological follow-up, however, disclosed increasing opacification on the left side with displacement of the heart and mediastinum to the left, as in atelectasis. Bronchoscopic examination revealed a narrowing of the distal end of the left main bronchus. There was a mucosal swelling and some bloody mucus in the lumen. An operation was considered but was postponed because the clinical condition improved and the chest film showed reexpansion of the lung. In March 1966, however, an operation was performed because a further bronchoscopy showed unmistakable stenosis of the left main bronchus.

This operation (performed elsewhere) involved excision of the stenosis followed by end-to-end anastomosis. It is important to note that the operation report mentioned torsion at the site of the anastomosis, observed at the end of the operation. The torsion was not corrected. The immediate postoperative course was uneventful, but a bronchoscopic follow-up after six weeks revealed a recurrence of stenosis at the site of the anastomosis. Initial measures were conservative-control of the bronchial infection and dilatation of the stenosis under bronchoscopic control. The bronchogram (Fig. 1) disclosed a stenosis localized to the left main bronchus, about $1.5 \mathrm{~cm}$ distal to the tracheal carina. Unmistakable post-stenotic bronchial dilatation was visible ; the duration of these changes was uncertain because no earlier bronchogram had been obtained.

In February 1967, one year after injury, the patient was transferred to our department for further treatment. The principal symptoms were cough, expectoration of purulent sputum, and dyspnoea. Alcaligenes faecalis was identified in the sputum. Bronchoscopic examination revealed a marked fibrous 


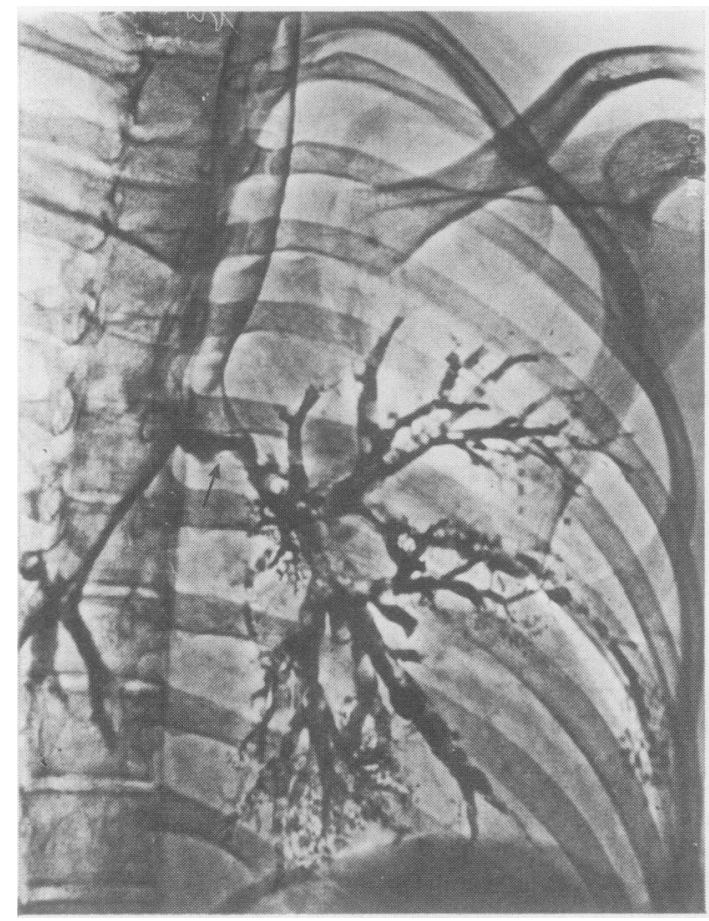

FIG. 1. Preoperative bronchogram (March 1966) shows post-stenotic dilatation of the lower lobe branches. Proximal to the stenosis (arrow) pooling of contrast medium is visible.

T A B L E I

SPIROMETRIC RESULTS

\begin{tabular}{|c|c|c|c|}
\hline & \multicolumn{2}{|c|}{ Observed } & \multirow{2}{*}{ Predicted } \\
\hline & $\begin{array}{l}\text { Preoperative } \\
28 \text { Fet. } 1967\end{array}$ & $\begin{array}{l}\text { Postoperative } \\
22 \text { Sept. } 1969\end{array}$ & \\
\hline $\begin{array}{ll}\text { VC (litres) } & \ldots \\
\mathrm{FEV}_{1.0} /(\% \text { VC) } & \cdots \\
\mathrm{MBC}(\text { litres/min) } & \ldots\end{array}$ & $\begin{array}{l}2 \cdot 66 \\
72 \cdot 5 \\
52 \cdot 8\end{array}$ & $\begin{array}{l}3 \cdot 51 \\
86 \\
81 \cdot 3\end{array}$ & $\begin{array}{l}3 \cdot 38 \\
70 \\
71\end{array}$ \\
\hline
\end{tabular}

stenosis (diameter about $0.5 \mathrm{~cm}$ ) $1.5 \mathrm{~cm}$ below the tracheal carina. With the aid of a thin catheter, mucopurulent material was obtained from the bronchial tree distal to the stenosis.

After preparation with antibiotics and physiotherapy, an operation was performed in March 1967. Breakdown of the earlier anastomosis was found. The distal and proximal bronchial ends were joined by a fibrous strand with a lumen of $3.0 \mathrm{~mm}$ diameter. This strand was excised and the distal and proximal bronchial ends were re-united by another end-to-end anastomosis using nylon sutures.

The postoperative course was uneventful. The radiological follow-up showed adequate expansion of the left lung. At follow-up two years later, the patient

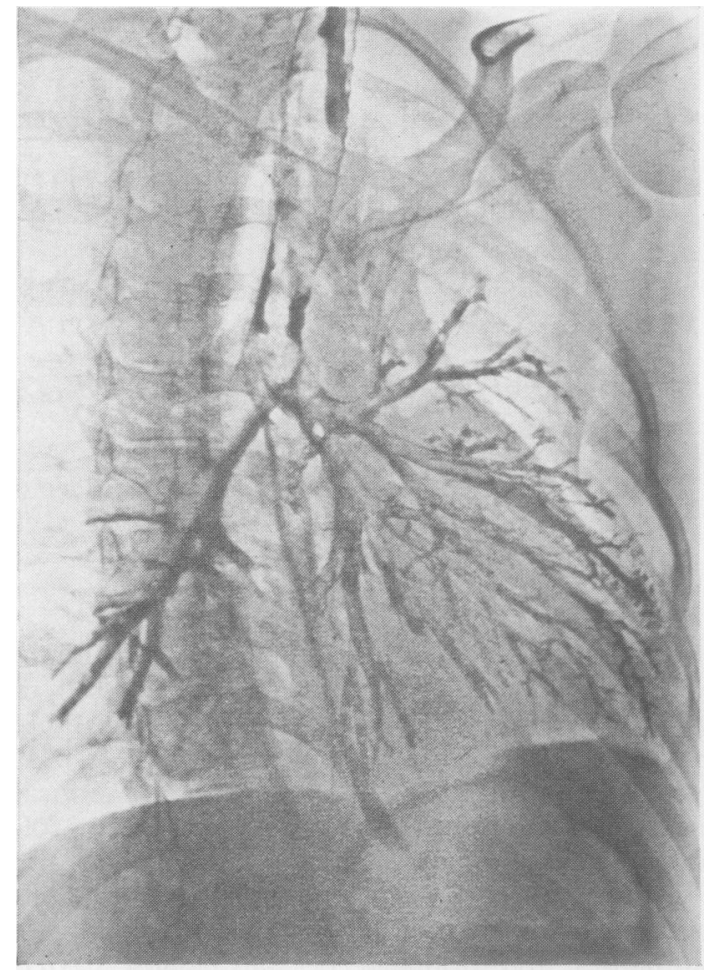

FIG. 2. Postoperative bronchogram (April 1968). The poststenotic bronchial dilatation has largely disappeared.

T A B L E I I BRONCHOSPIROMETRIC RESULTS

\begin{tabular}{|c|c|c|c|}
\hline & \multicolumn{3}{|c|}{ Postoperative 17 Apr. 1968} \\
\hline & Total & Right $\%$ & Left $\%$ \\
\hline $\begin{array}{l}\text { VC (litres) } \\
\mathrm{O}_{2} \text { consumption (litres/min) } \\
\text { Tidal volume (litres) }\end{array}$ & $\begin{array}{l}2 \cdot 96 \\
0 \cdot 32 \\
0 \cdot 40\end{array}$ & $\begin{array}{l}59 \\
62 \cdot 5 \\
57\end{array}$ & $\begin{array}{l}41 \\
37 \cdot 5 \\
43\end{array}$ \\
\hline
\end{tabular}

was free from symptoms. The bronchogram (Fig. 2) showed only slight changes, and bronchoscopic exam- 을 ination revealed a fibrous ring at the site of the $\mathrm{N}$ anastomosis without noticeable stenosis. Lung func- $\sigma$ tion was virtually normal (Tables I and II).

\section{DISCUSSION}

Several reports (Richards and Cohn, 1955; Isfort 1967; Truckenbrodt and Gall, 1968) maintain that surgical repair of the bronchus after post- $\stackrel{?}{?}$ traumatic bronchial stenosis is of use only if there $\bar{T}$ is no distal bronchial dilatation. This opinion is emphasized when chronic infection of the lung exists. 
Drapanas et al. (1966) described a patient in whom a thoracic injury resulted in bronchial stenosis with post-stenotic bronchiectasis. This change was already present 26 days after the accident. A few days later, surgical repair of the bronchus was successfully carried out and the bronchiectatic changes resolved.

The literature contains occasional similar case reports. Uncertainty exists as to how long one should wait, when to perform a bronchoplastic operation, and when to resort to lobectomy or pneumonectomy. Our case report supports our belief that post-stenotic bronchial dilatation or bronchiectasis of some standing, infected or not, need be no contraindication to a bronchoplastic operation.

\section{REFERENCES}

Drapanas, T., Siewers, R., and Feist, J. H. (1966). Reversible poststenotic bronchiectasis. New Engl. J. Med., 275, 917.

Eijgelaar, A., and Homan van der Heide, J. N. (1970). A reliable early symptom of bronchial or tracheal rupture. Thorax, 25, 120.

Isfort, A. (1967). Traumatische Bronchusstenose mit Bronchiektasen. Thoraxchirurgie, 15, 653.

Richards, V., and Cohn, R. B. (1955). Rupture of the thoracic trachea and major bronchi following closed injury to the chest. Amer. J. Surg., 90, 253.

Truckenbrodt, H., and Gall, F. (1968). Zur Diagnose und Therapie der Bronchusruptur. Radiologe, 8, 315. 